\title{
Risk Factors for the Development of Esophagorespiratory Fistula in Esophageal Cancer
}

\author{
Gregor Paul, Wolfram Bohle, Wolfram Zoller
}

Department of General

Internal Medicine

and Gastroenterology,

Katharinenhospital, Stuttgart, Germany

\author{
Address for correspondence: \\ Gregor Paul \\ Department of General \\ Internal Medicine \\ and Gastroenterology, \\ Katharinenhospital \\ Kriegsbergstraße 60, \\ 70174 Stuttgart, Germany \\ gr.paul@klinikum-stuttgart.de
}

Received: 13.07.2019

Accepted: 11.08.2019

\begin{abstract}
Background \& Aims: The development of esophagorespiratory fistula (ERF) in esophageal cancer (EC) is a devastating complication, leading to poor survival rates and low quality of life. The aim of this study was to identify the risk factors leading to fistula formation in EC.

Methods: We identified 47 patients with malignant ERF formation in EC in a period of 10 years. Clinical characteristics were compared by univariable analysis with 47 randomly selected patients with EC without ERF. A case-control study was conducted for patients with squamous cell carcinoma (SCC) and ERF matching in a 1:2 fashion for primary tumor location.

Results: The identified risk factors in EC patients were histology of SCC ( $<<0.001)$, former or current smoking status ( $\mathrm{p}=0.002)$ and primary tumor location in the proximal esophagus $(\mathrm{p}<0.001)$. The „hot spot” for ERF formation was tumor growth $20-25 \mathrm{~cm}$ distal to dental arch. An additional risk factor in SCC patients was age. Patients with ERF formation in SCC were younger than patients without ERF (median 63 vs. 67 years, $p=0.02$ ). No difference in the rate of fistula formation was seen between esophagectomy and definitive chemoradiation, but the latter developed ERF earlier in the course of the disease ( $237 \mathrm{vs.} 596.5$ days, $\mathrm{p}=0.01$ ). Conclusion: Patients with proximal SCC of the esophagus and a smoking history, as well as young patients with SCC should be closely monitored for ERF formation.
\end{abstract}

Key words: esophagorespiratory fistula - esophageal cancer - smoking - risk factors.

Abbreviations: EC: esophageal cancer; ERF: esophagorespiratory fistula; SCC: squamous cell carcinoma (SCC).

\section{INTRODUCTION}

The development of esophagorespiratory fistulas (ERF) is usually a late complication in patients with esophageal cancer (EC). Esophageal cancer is the most common cause of ERF formation and develops in $5-15 \%$ of the patients [1]. Anatomically, the esophagus lies in close proximity to the trachea, bronchi, lung, aorta, left atrium and spine. A communication between the esophagus and the airways may result by direct propagation of the tumor or is a consequence of side effects of treatment, especially radiotherapy [2]. The risk factors leading to the development of ERF in esophageal cancer are only scarcely investigated. Known risk factors are advanced stage of disease, involvement of upper-mid thoracic esophagus, length of primary tumor, complete circumferential lesions and esophageal stenosis [3-5]. Patients with ERF have high morbidity and mortality rates, as a consequence of perpetual aspiration and pulmonary infection [6]. Implantation of selfexpandable metal stents in the esophagus, the trachea or both, is the treatment of choice [7]. On the other hand, implantation of metal stents itself can lead to fistula formation, as a result of compression and wall necrosis of the esophagus [8]. Sealing malignant ERF with either esophageal or airway stents has shown to improve overall quality of life [9].

The aim of this study was to screen for additional risk factors for ERF, especially in locally advanced tumors. More potential risk factors need to be discovered, so patients with a high risk of ERF formation can be identified earlier in the course of the disease, thereby either preventing fistula formation or delaying its consequences. 


\section{METHODS}

\section{Patients}

Forty-seven adult patients with ERF in EC were identified by screening the local patient record database at the Klinikum Stuttgart for the period 2008-2017. Multiple databases, such as the institutional endoscopy database and databases containing discharge diagnosis were used. Esophagorespiratory fistula was diagnosed by either direct visualization during gastroscopy and bronchoscopy or contrast radiography of the upper gastrointestinal tract.

The study was approved and authorized by the hospital Ethics Committee of the University of Tübingen, project number: 879/2017BO2. Since diagnosis and treatment correspond to the quality standard of current guidelines, no written informed consent of the patients was considered necessary.

\section{Data acquisition}

Information recorded was: age at initial diagnosis, gender, location of tumor (proximal, middle, distal part of esophagus and distance in centimeters from the dental arch), length of tumor as measured by endoscopy, histology (squamous vs. non-squamous), grading (1-3), initial tumor stage (T1-4), node stage ( $\mathrm{N} 0$ or $\mathrm{N}+$ ), or metastasis stage (M0 or M1), former or current smoking status, body mass index (BMI), body height, relapse, former or current tumor other than EC and need for bougienage. TNM information was documented by imaging studies, endoscopy or endoscopic ultrasound (EUS). Proximal esophagus was defined as majority of tumor growth up to 25 $\mathrm{cm}$ from incisors, middle as $25-32 \mathrm{~cm}$ from incisors and distal from $32 \mathrm{~cm}$ and beyond. Not all variables were readily available in all patients in the database, leading to partly missing cases. If patients had satellite tumors, only the main tumor, i.e. the tumor that led to fistula formation was appreciated.

\section{Distribution}

For representation, a "model esophagus" was assumed with a length of 13-40 cm from the incisors. Per centimeter, the number of tumors that affected this region was counted. For instance, a tumor growing from 22 to $24 \mathrm{~cm}$ from incisors would therefore be represented at 22, 23 and $24 \mathrm{~cm}$. The results were displayed in a bar graph, generated with GraphPad Prism Version 7. Results are represented as mean in centimeters plus standard deviation. A t-test was applied for statistical analysis.

\section{Statistical analysis}

Forty-seven patients with ERF were compared with 47 randomly selected patients with EC, but without ERF. Random selection was performed by software based random allocation. Characteristics of the patients with or without ERF were compared using Fisher's exact test for categorical variables and Student $t$-test for metric variables. In a second step, 43 patients with squamous cell carcinoma (SCC) and ERF were matched, in a 1:2 fashion, to 86 randomly selected controls for tumor localization. Controls were selected from the total cohort of 554 patients with EC. Matching was performed by MedCalc Version 19.0.5. A case-control design was used to identify risk factors for ERF formation, independent of tumor location and histology. Because a matched dataset was used for the analysis, conditional logistic regression analysis was conducted to estimate the odd ratios (ORs) and 95\% confidence intervals (CIs) and to assess the association between clinical characteristics and ERF formation. Covariates were selected through a backward stepwise selection. A p-value of $<0.05$ was considered statistically significant. The statistical analysis was conducted using IBM SPSS Statistics Version 24.

\section{RESULTS}

\section{Clinical characteristics}

In a period of 10 years, 47 out of 554 patients with EC developed ERF. This correlates to a cumulative incidence of $8.4 \%$. Thirty-five patients ( $74.5 \%)$ had fistula formation to the trachea, $10(21.3 \%)$ to the left main bronchus and $2(4.2 \%)$ to the right main bronchus. Median time from initial diagnosis to ERF formation was 253 days (range 0-2061 days). Patients after definitive chemoradiotherapy developed ERF earlier (median 237 days, range 31-1065) than patients after esophagectomy (596.5 days, range 31-1696) ( $\mathrm{p}=0.01$ ).

Four patients presented with ERF at initial diagnosis, while 19 patients with ERF developed this complication as a pattern of disease recurrence. Out of these 19 patients, 10 had had a previous esophagectomy and 9 patients definitive chemoradiotherapy. Twenty-four patients developed an ERF progressively during the course of disease, 11 out of 24 patients developed ERF during definitive chemoradiotherapy, 7 patients during palliative chemotherapy, 3 patients had radiotherapy and 3 patients received no treatment at all.

Forty-two patients with ERF had stent placement either in the esophagus or in the trachea. In 28 patients, double stenting in the esophagus and trachea was performed. Eleven patients had solely an esophageal stent and 3 patients solely a tracheal stent.

\section{Risk factors for ERF formation in patients with EC}

Median age in the control group without ERF in EC was 64 years (range 40-86) versus 61 years (45-82) for patients with ERF. Gender distribution was $83 \%$ male and $17 \%$ female in the control group, compared to $81 \%$ and $19 \%$ in the ERF group. There was no significant difference regarding age or gender distribution between groups. Table I shows that patients with ERF formation were more likely to have SCC ( $p \leq 0.001)$ and were more likely to be former or current smokers ( $\mathrm{p}=$ 0.002). Synchronous cancer or a history of a previous tumor other than EC was not associated with a higher risk for fistula formation. One patient in the control group had synchronous oral cancer. Twelve patients had a history of cancer other than EC (5 malignant ENT-tumors, 2 prostate, testicular, breast, duodenal, renal and hepatic cancer). Two patients in the ERF group had synchronous cancer, namely one lung cancer and one hypopharyngeal cancer. Nine patients had a past history of cancer (5 malignant ENT-tumors, prostate, breast, cervical and colon cancer).

\section{Distribution}

Tumors that led to ERF formation affected more often the proximal part $(p \leq 0.001)$ and less often the distal part of the 
Table I. Risk factors of esophagorespiratory fistula formation in patients with esophageal cancer and controls

\begin{tabular}{|c|c|c|c|c|}
\hline & $\begin{array}{l}\text { No fistula } \\
(\mathrm{N}=47)\end{array}$ & $\begin{array}{l}\text { Fistula } \\
(\mathrm{N}=47)\end{array}$ & Odds ratio & p-value \\
\hline Median age (range) & $64(40-86)$ & $61(45-82)$ & - & 0.25 \\
\hline \multicolumn{5}{|l|}{ Gender n (\%) } \\
\hline Female & $8(17)$ & $9(19)$ & & \\
\hline Male & $39(83)$ & $38(81)$ & $0.97(0.332-2.86)$ & 1 \\
\hline \multicolumn{5}{|l|}{ Histology n (\%) } \\
\hline Non SCC & $19(40)$ & $4(9)$ & & \\
\hline SCC & $28(60)$ & $43(91)$ & $9.5(2.57-35.15)$ & $<0.001$ \\
\hline \multicolumn{5}{|l|}{ T stage n (\%) } \\
\hline Non T4 & $32(70)$ & $31(66)$ & & \\
\hline $\mathrm{T} 4$ & $14(30)$ & $16(34)$ & $1.22(0.51-0.7)$ & 0.82 \\
\hline \multicolumn{5}{|l|}{$\mathrm{N}$ stage $\mathrm{n}(\%)$} \\
\hline No & $6(14)$ & $9(19)$ & & \\
\hline $\mathrm{N}+$ & $37(86)$ & $36(81)$ & $0.63(0.2-1.96)$ & 0.57 \\
\hline \multicolumn{5}{|l|}{ M stage n (\%) } \\
\hline M0 & $34(74)$ & $32(68)$ & & \\
\hline M1 & $12(26)$ & $14(32)$ & $1.28(0.51-3.19)$ & 0.65 \\
\hline \multicolumn{5}{|l|}{ Grading n (\%) } \\
\hline $\mathrm{G} 1+\mathrm{G} 2$ & $22(49)$ & $23(55)$ & & \\
\hline G3 & $23(51)$ & $19(45)$ & $0.83(0.35-1.93)$ & 0.67 \\
\hline \multicolumn{5}{|l|}{ Tumor growth n (\%) } \\
\hline Proximal segment & $12(25.5)$ & $32(68)$ & $6.03(2.45-14.83)$ & $<0.001$ \\
\hline Middle segment & $12(25.5)$ & $13(27.7)$ & $1.15(0.46-2.88)$ & 0.82 \\
\hline Distal segment & $23(49)$ & $2(4.3)$ & $0.13(0.04-0.38)$ & $<0.001$ \\
\hline \multicolumn{5}{|l|}{ Smoking n (\%) } \\
\hline Non-Smoker & $24(51)$ & $9(19)$ & & \\
\hline Smoker & $23(49)$ & $38(81)$ & $4.29(1.7-10.83)$ & 0.002 \\
\hline \multicolumn{5}{|l|}{ Previous tumor $\mathrm{n}(\%)$} \\
\hline No tumor & $34(72)$ & $36(77)$ & & \\
\hline Previous tumor & $13(28)$ & $11(23)$ & $0.726(0.28-1.88)$ & 0.63 \\
\hline $\begin{array}{l}\text { Tumor mean length, } \mathrm{cm} \\
\text { (standard deviation) }\end{array}$ & $4.8( \pm 2.9)$ & $5.1( \pm 2.1)$ & - & 0.65 \\
\hline
\end{tabular}

esophagus ( $p \leq 0.001)$. No difference was seen for the middle segment $(\mathrm{p}=0.82)$. Fig. 1 shows the distribution of tumor location, as measured in centimeters from the incisors. Tumor location was at $27 \mathrm{~cm}(\mathrm{SD} \pm 6.9 \mathrm{~cm})$ for the control group and $24.2 \mathrm{~cm}(\mathrm{SD} \pm 4.4 \mathrm{~cm})$ for the ERF group. Median of the tumor location was $27 \mathrm{~cm}$ (range 13-40 cm) for control and $24 \mathrm{~cm}$ (16-35 cm) for ERF group. The distribution was statistically different $(\mathrm{p} \leq 0.001)$. Tumors that led to ERF formation showed no growth below $16 \mathrm{~cm}$ and beyond $36 \mathrm{~cm}$ from the incisors. As seen in the graph, more tumors that led to ERF formation showed growth 20-25 cm from the incisors (Fig. 1).

Risk factors for ERF formation in patients with SCC of the esophagus

The two strongest risk factors for ERF formation in EC patients were a histology of SCC and tumor growth in the proximal part of the esophagus. To identify risk factors of ERF formation that were independent of histology and tumor localization, we performed a case-control study, matching all 43 patients with SCC and ERF in a 1:2 fashion to controls for tumor location (proximal, middle and distal esophagus). The control group comprised 56 patients with proximal EC, 26 with middle and 4 with distal SCC without ERF. In the ERF group there were 28 patients with proximal, 13 with middle and 2 with distal SCC and ERF. Median age in the control group was 67 years (45-87) versus 63 years (45-82) in patients with ERF. Gender distribution was $76 \%$ male and $24 \%$ female in the control group and $79 \%$ male and $21 \%$ female in the ERF group. Patients with ERF in SCC were younger than patients without fistula formation ( $\mathrm{p}=0.02$ ) (Table II). No other risk factor reached statistical significance by univariable analysis.

The multivariate analysis was performed after adjusting for tumor location. There was a significant effect of a younger age on the occurrence of ERF in the study patients $(p=0.03)$. No other factor reached statistical significance by multivariate analysis. 


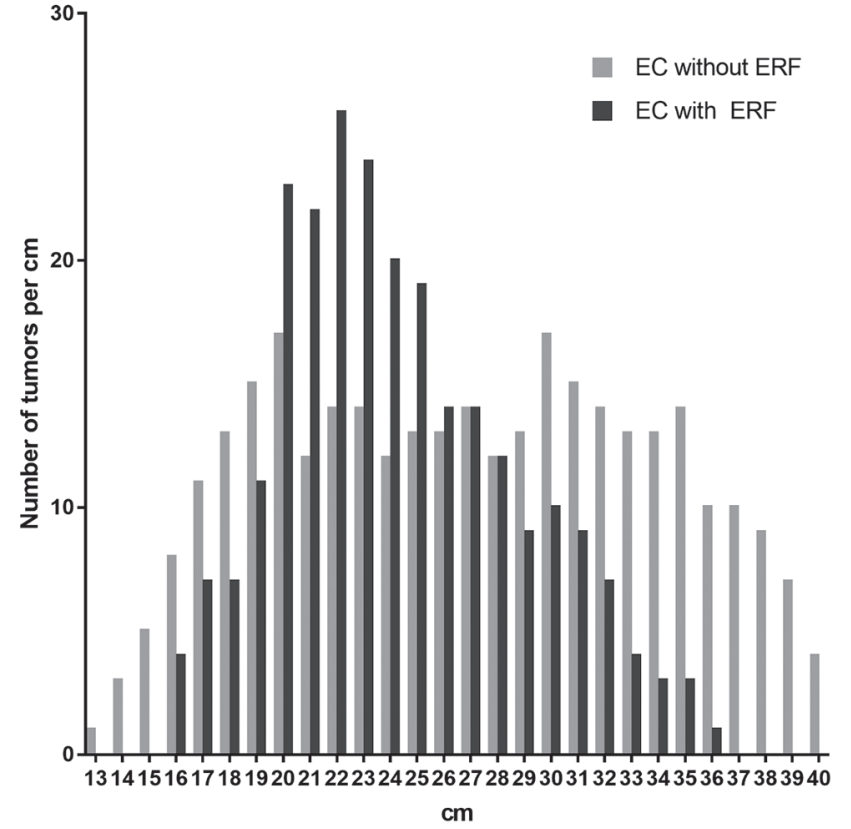

Fig. 1. Distribution of tumor localization, as measured in centimeters from the incisors (X-axis). Y-axis shows the number of tumors per $\mathrm{cm}$. Abbreviations: EC:esophageal cancer; ERF: esophagorespiratory fistula.

\section{DISCUSSION}

Proximal tumor growth, SCC and smoking are the risk factors for ERF formation in EC identified in our study. Smoking has only rarely been studied as a potential risk factor for ERF formation. The most probable explanation for its association with ERF is that tobacco abuse is directly linked to SCC development. Around $49 \%$ of all esophageal SCC cases can be attributed to smoking [10]. Since SCCs most commonly occur in the proximal and middle third of the esophagus, smoking might simply raise the risk for ERF formation in EC by raising the risk for SCC. This is corroborated by the fact that smoking was not an identifiable risk factor in our case-control approach in which only patients with SCC were compared. The group by Zhang et al. [11] also evaluated smoking as a risk factor for ERF formation in SCC patients. No association was detected in their final multivariate analysis, which is in accordance with our data.

We showed that tumor growth between $20-25 \mathrm{~cm}$ from the incisors is a "hot spot" for fistula formation. This is in agreement with a study by Bick et al. [12], which showed that most esophageal lesions that led to ERF formation, before and after esophageal stent placement, were situated in-between $21-25 \mathrm{~cm}$ from the incisors. This might be explained by the

Table II. Risk factors of ERF formation in esophageal squamous cell carcinoma

\begin{tabular}{|c|c|c|c|c|}
\hline & $\begin{array}{l}\text { No fistula } \\
(\mathrm{N}=86)\end{array}$ & $\begin{array}{l}\text { Fistula } \\
(\mathrm{N}=43)\end{array}$ & Odds ratio & p-value \\
\hline Median age (range) & $67(45-87)$ & $63(45-82)$ & - & 0.02 \\
\hline \multicolumn{5}{|l|}{ Gender n (\%) } \\
\hline Female & $21(24)$ & $9(21)$ & & \\
\hline Male & $65(76)$ & $34(79)$ & $1.22(0.54-2.96)$ & 0.83 \\
\hline \multicolumn{5}{|l|}{ Chemotherapy n (\%) } \\
\hline No chemotherapy & $9(11)$ & $8(19)$ & & \\
\hline Chemotherapy & $77(89)$ & $34(81)$ & $0.5(0.18-1.4)$ & 0.27 \\
\hline \multicolumn{5}{|l|}{ Radiotherapy n (\%) } \\
\hline No radiotherapy & $22(26)$ & $14(33)$ & & \\
\hline Radiotherapy & $64(74)$ & $28(67)$ & $0.69(0.31-1.54)$ & 0.41 \\
\hline \multicolumn{5}{|l|}{ Surgery n (\%) } \\
\hline No surgery & $72(84)$ & $34(79)$ & & \\
\hline Surgery & $14(16)$ & $9(21)$ & $1.36(0.54-3.6)$ & 0.63 \\
\hline \multicolumn{5}{|l|}{ Bougienage n (\%) } \\
\hline No bougienage & $34(40)$ & $13(31)$ & & \\
\hline Bougienage & $52(60)$ & $29(69)$ & $1.46(0.67-3.2)$ & 0.44 \\
\hline \multicolumn{5}{|l|}{ Relapse n (\%) } \\
\hline No relapse & $57(66)$ & $25(60)$ & & \\
\hline Relapse & $29(34)$ & $17(41)$ & $1.34(0.62-2.86)$ & 0.56 \\
\hline \multicolumn{5}{|l|}{ Smoking n (\%) } \\
\hline Non-Smoker & $21(25)$ & $8(19)$ & & \\
\hline Smoker & $64(75)$ & $35(81)$ & $1.44(0.58-3.57)$ & 0.5 \\
\hline $\begin{array}{l}\text { Body height, } \mathrm{cm} \\
\text { (standard deviation) }\end{array}$ & $171( \pm 8.5)$ & $173( \pm 9.5)$ & - & 0.34 \\
\hline $\begin{array}{l}\mathrm{BMI}, \mathrm{kg} / \mathrm{m}^{2} \\
\text { (standard deviation) }\end{array}$ & 22.8 & 21.6 & - & 0.19 \\
\hline
\end{tabular}

BMI: body mass index 
anatomical proximity of the trachea to the esophagus in this region. The distance of the pars membranacea of the trachea to the esophagus is often less than $0.5 \mathrm{~mm}$ for a distance of 7-8 $\mathrm{cm}$ in the posterior mediastinum. After division into the left and right main bronchi the distance extends to $3 \mathrm{~mm}$ between the esophagus and the left main bronchus. The distance to the right main bronchus is even bigger, as a result of a slight left side deviation of the esophagus [13]. This explains the fact why the majority of patients, i.e. $35(74.4 \%)$ had a fistula to the trachea. Ten patients $(21.3 \%)$ showed fistula formation to the left main bronchus and only 2 patients (4.3\%) to the right main bronchus. Tumor location and histology, namely SCC, are the strongest risk factors for fistula formation. To identify risk factors that have a smaller impact on ERF formation, we performed a case-control study in patients with esophageal SCC, matching for the variable tumor location. We found that patients with SCC and ERF were significantly younger than patients with SCC without ERF. A similar observation was reported by Choi et al. [3] and by Balazs et al. [14]. The age-specific risk factors for SCC are not well explained. One might hypothesize that younger age at disease onset is a result of a more intense exposure to tobacco smoking and alcoholic beverages. This is in accordance with the observation that ERF formation is associated with the histology of SCC, for which smoking and alcohol are the two main risk factors.

In our study, we were not able to correlate any therapeutic strategy, such as chemotherapy, radiotherapy or surgery to ERF formation. Previous studies were able to show that the development of ERF is up to 9.4-fold more common after esophageal stent placement in patients that underwent prior radiation therapy, with or without chemotherapy $[3,12]$. As we did not discern between patients with and without esophageal stent placement prior or post radiation therapy, we were not able to detect a significant difference. However, our data evidenced that patients undergoing definitive chemoradiation develop ERF earlier in the course of the disease (237 vs. 596.5 days). Radiotherapy is known to cause esophageal inflammation and tumor necrosis and might thereby facilitate early ERF formation [15]. Zhang et al. [11] evaluated the fraction dose of radiotherapy as a risk factor for fistula formation. While in univariate analysis the fraction dose was associated with fistula formation, the results were not significant in multivariate analysis [11]. Tsushima et al. [4] found no difference for the rate of fistula formation between the group of patients receiving standard dose cisplatin +5 -fluorouracil and the group with low dose cisplatin +5 -fluorouracil.

The nutritional status has also been reported to influence fistula formation after radiotherapy. Watanabe et al. [16] found that patients with a BMI below $20 \mathrm{~kg} / \mathrm{m}^{2}$ were at higher risk for ERF formation after radiotherapy. Nutritional status was not associated with ERF formation in our study, most likely because we did not perform subgroup analysis for patients with radiotherapy.

Our study is limited by a small sample size and its retrospective approach. Because ERF formation is a rather infrequent complication in esophageal cancer, it would have been difficult to perform a prospective trial. Thereby, data quality relies on the accurate documentation in the past.

\section{CONCLUSIONS}

Tumor growth in the proximal part of the esophagus, smoking and histology of SCC were strong risk factors for ERF formation in EC. Definitive chemoradiotherapy facilitated early ERF formation compared to esophagectomy, but was not an independent risk factor in our patient population. In clinical practice, more attention should be given to young patients with EC, with a history of smoking and proximal tumor growth. Thus, patients at high risk for ERF could be identified earlier in the course of the disease.

\section{Conflicts of interest: None to declare.}

Authors' contributions: G.P. collected and analyzed the data, designed the study and prepared the manuscript. W.B. and W.Z. reviewed the manuscript and contributed to data collection. All authors critically revised the manuscript and approved the final version.

\section{REFERENCES}

1. Spaander MC, Baron TH, Siersema PD, et al. Esophageal stenting for benign and malignant disease: European Society of Gastrointestinal Endoscopy (ESGE) Clinical Guideline. Endoscopy 2016;48:939-948. doi:10.1055/s-0042-114210

2. Gore E, Currey A, Choong N. Tracheoesophageal fistula associated with bevacizumab 21 months after completion of radiation therapy. J Thorac Oncol 2009;4:1590-1591. doi:10.1097/JTO.0b013e3181c06a6f

3. Choi MK, Park YH, Hong JY, et al. Clinical implications of esophagorespiratory fistulae in patients with esophageal squamous cell carcinoma (SCCA). Med Oncol 2010;27:1234-1238. doi:10.1007/ s12032-009-9364-Z

4. Tsushima T, Mizusawa J, Sudo K, et al. Risk Factors for Esophageal Fistula Associated With Chemoradiotherapy for Locally Advanced Unresectable Esophageal Cancer: A Supplementary Analysis of JCOG0303. Medicine (Baltimore) 2016;95:e3699. doi:10.1097/ MD.0000000000003699

5. Kawakami T, Tsushima T, Omae K, et al. Risk factors for esophageal fistula in thoracic esophageal squamous cell carcinoma invading adjacent organs treated with definitive chemoradiotherapy: a monocentric case-control study. BMC Cancer 2018;18:573. doi:10.1186/ s12885-018-4486-3

6. Ribeiro MSI, da Costa Martins B, Simas de Lima M, et al. Selfexpandable metal stent for malignant esophagorespiratory fistula: predictive factors associated with clinical failure. Gastrointest Endosc 2018;87:390-396. doi:10.1016/j.gie.2017.09.020

7. Chen YH, Li SH, Chiu YC, et al. Comparative Study of Esophageal Stent and Feeding Gastrostomy/Jejunostomy for Tracheoesophageal Fistula Caused by Esophageal Squamous Cell Carcinoma. PLoS One 2012;7:e42766. doi:10.1371/journal.pone.0042766

8. Ke M, Wu X, Zeng J. The treatment strategy for tracheoesophageal fistula. J Thorac Dis 2015;7Suppl 4:S389-S397. doi:10.3978/j.issn.20721439.2015.12.11

9. Herth FJ, Peter S, Baty F, Eberhardt R, Leuppi JD, Chhajed PN Combined airway and oesophageal stenting in malignant airwayoesophageal fistulas: a prospective study. Eur Respir J 2010;36:13701374. doi:10.1183/09031936.00049809 
10. Pandeya N, Olsen CM, Whiteman DC. Sex differences in the proportion of esophageal squamous cell carcinoma cases attributable to tobacco smoking and alcohol consumption. Cancer Epidemiol 2013;37:579-584. doi:10.1016/j.canep.2013.05.011

11. Zhang Y, Li Z, Zhang W, Chen W, Song Y. Risk factors for esophageal fistula in patients with locally advanced esophageal carcinoma receiving chemoradiotherapy. Onco Targets Ther 2018;11:2311-2317. doi:10.2147/OTT.S161803

12. Bick BL, Song LM, Buttar NS, et al. Stent-associated esophagorespiratory fistulas: incidence and risk factors. Gastrointest Endosc 2013;77:181189. doi:10.1016/j.gie.2012.10.004

13. Liebermann-Meffert D. Anatomical basis for the approach and extent of surgical treatment of esophageal cancer. Dis Esophagus 2001;14:81-84. doi:10.1046/j.1442-2050.2001.00160.x
14. Balazs A, Galambos Z, Kupcsulik PK. Characteristics of esophagorespiratory fistulas resulting from esophageal cancers: a single-center study on 243 cases in a 20 -year period. World J Surg 2009;33:994-1001. doi:10.1007/s00268-009-9988-3

15. Kinsman KJ, DeGregorio BT, Katon RM, et al. Prior radiation and chemotherapy increase the risk of life-threatening complications after insertion of metallic stents for esophagogastric malignancy. Gastrointest Endosc 1996;43:196-203. doi:10.1016/s00165107(96)70315-2

16. Watanabe S, Ogino I, Kunisaki C, Hata M. Relationship between nutritional status and esophageal fistula formation after radiotherapy for esophageal cancer. Cancer Radiother 2019;23:222-227. doi:10.1016/j. canrad.2018.10.005 